\title{
Anesthetic Management in Bilateral Deep Brain Stimulation for X-linked Dystonia Parkinsonism: Early Single Institution Experience from the Philippines
}

\author{
Mary Ellen Chiong-Perez, ${ }^{1,2}$ Cid Czarina E. Diesta² and Jean Quint L. Oropilla² \\ ${ }^{1}$ Department of Anesthesiology, College of Medicine and Philippine General Hospital, University of the Philippines Manila \\ ${ }^{2}$ Asian Hospital and Medical Center
}

\begin{abstract}
X-linked dystonia-parkinsonism (XDP) is a rare, adult-onset, progressive, hereditary neurological movement disorder primarily affecting Filipino men with maternal families from Panay province of the Philippines. Medical treatment modalities currently being used have offered temporary symptomatic relief. Surgical management in the form of bilateral globus pallidi internae (Gpi) deep brain stimulation (DBS) has shown promising results and is increasingly being performed in advanced centers, as reported in international literature.

Presented herein is the local experience of seven (7) retrospectively reviewed cases from February 2018 to February 2019 in a tertiary center in the Philippines with a particular focus on anesthetic management.

All patients were male, from Panay, and presented with progressive dystonia and parkinsonism. All patients underwent planned bilateral, simultaneous DBS electrode, and implantable pulse generator (IPG) placement performed by a multidisciplinary team. Anesthetic management consisted of Bispectral Index (BIS) guided conscious sedation with low dose propofol and remifentanil infusions with a complete scalp nerve block (SB) at the start of the procedure then shifted to awake monitored anesthesia care during electrode placement, microelectrode recording (MER) and macro stimulation testing. All were put under general anesthesia with a supraglottic airway device during the placement of the internal pulse generator (IPG) in the infraclavicular area. All seven patients had successful localization, and insertion of the DBS electrode and discharged improved. The anesthetic management of the DBS used in these cases warrants further investigation and may lead to standardization of future practice.
\end{abstract}

Key Words: X-linked dystonia-parkinsonism, deep brain stimulation, anesthetic management

\section{INTRODUCTION}

$\mathrm{X}$-linked dystonia-parkinsonism (XDP), also known as Lubag Disease, is a hereditary, neurodegenerative disorder primarily affecting Filipino men with maternal roots from the Panay province of the Philippines. It is an adult-onset, severe, progressive movement disorder, characterized by a wide array of neurological symptoms. The usual initial presentation includes focal dystonia such as blepharospasm, oromandibular, lingual or pharyngeal dystonia, torticollis, myorhythmia, tremor, or dystonia of a limb. Within five to six years, symptoms often progress to multifocal or generalized dystonia that combines with or replaced by parkinsonism. These can result in poor quality of life due to pain (in cervical

Corresponding author: Mary Ellen Chiong-Perez, MD Department of Anesthesiology Philippine General Hospital

University of the Philippines Manila

Taft Avenue, Manila 1000, Philippines

Email: mcperez6@up.edu.ph dystonia and contractures), immobility, insufficient food intake, weight loss, aspiration, and respiratory stridor, the latter two being the most common cause of death among these patients. ${ }^{1}$ Medical treatment modalities, such as chemodenervation with botulinum toxin injection or oral 
anti-dystonic drugs such as anticholinergics, baclofen, and clonazepam, have modest benefits with reported side effects or does not confer significant improvement from baseline, such as in treatment with levodopa+carbidopa. ${ }^{2}$

Reports have shown that surgical management in the form of bilateral Globus pallidi internae (GPi) deep brain stimulation improves the symptoms in varying degrees. It represents a significant major therapeutic advance for parkinsonism and dystonia, including XDP. Although several reports have been published regarding the increasing experience with DBS for XDP, the majority of the published literature focuses on the technical aspects of this neurosurgical procedure and the relevant patient outcomes. ${ }^{3,45}$ Likewise, although the disease is endemic in a Philippine province, most of the published experience in anesthetic management comes from foreign literature. ${ }^{6,7}$ This is probably due to the costs involved and the intensive technological requirements, making it inaccessible to most of those affected. ${ }^{8}$ Recent advances in knowledge and capability in the Philippine setting will hopefully address this gap. At the time of writing this report, there are only three centers in the Philippines that perform DBS. Of these, the Philippine Movement Disorder Surgery Center (PhilMove) has previously reported a series of nine XDP patients who had bilateral GPi-DBS (October 2009-September 2018), done either under local anesthesia or with dexmedetomidine as their primary sedative agent. ${ }^{9,10}$ Presented herein is a report of the GPiDBS cases performed in a private tertiary hospital in the Philippines with a particular focus on the various anesthetic management for the different phases of the procedure.

\section{OBJECTIVE}

The purpose of this report is to review our experience in the perioperative anesthetic management of patients with X-linked dystonia-parkinsonism (XDP) for deep brain stimulation (DBS) surgery. Specific attention to the techniques, choices of drugs, and anesthetic considerations for each phase of the surgery will be discussed.

The records of patients who underwent DBS for XDP in a single tertiary hospital in Metro Manila from February 2018 to February 2019 were reviewed. Patient demographics, disease presentation, diagnostic, and therapeutic history were extracted. Intraoperative data with particular emphasis on anesthesia technique were recorded, along with perioperative patient outcomes.

\section{Presentation of Cases}

Seven patients with XDP were admitted to our institution and underwent planned bilateral, simultaneous DBS electrode implantation and implantable pulse generator (IPG) placement from February 2018 to February 2019. All were Filipino males, all with maternal roots from Panay Island, with a mean age of 43 years $(\mathrm{SD}=8.8$, range of 31 to 59 years old) presenting with a history of slowly progressive dystonia and parkinsonism starting from 2 to 8 years before their admission to hospital (Table 1). All experienced a sense of agitation with extreme difficulty in relaxation. The diagnosis of XDP was confirmed genetically by the identification of disease-specific single-nucleotide changes of the TAF1 gene in all cases. Several pharmacological treatments were tried in all of them with minimal to no benefit. Chemodenervation with botulinum toxin injections were used in some patients for the neck and legs, but with suboptimal effectiveness. All were offered a surgical option for their conditions and consented to the procedure of bilateral GPi-DBS.

DBS per se is a multi-phase surgery. The first stage is the bilateral placement of electrodes into particular areas of the brain; in our case, the Gpi nuclei. The second stage involves the tunneling of the extension cable of the electrodes subcutaneously from the cranial area to the infraclavicular area where they are connected to the programmable Implantable Pulse Generator (IPG). Preparation for the actual surgery entails significant anesthesiologist participation.

Table 1. Demographics and Symptoms of the XDP Patients who underwent GPi-DBS in our center from February 2018 to February 2019

\begin{tabular}{|c|c|c|c|c|c|c|}
\hline Patient & Age/Sex & ASA status & Place of origin & Age at onset & Symptoms & Comorbidities \\
\hline 1 & $36 / M$ & 1 & Capiz & 32 & $\begin{array}{l}\text { Generalized dystonia, difficulty with jaw opening } \\
\text { and closing, twisted trunk }\end{array}$ & Psoriasis \\
\hline 2 & $46 / \mathrm{M}$ & 1 & Aklan & 43 & $\begin{array}{l}\text { Generalized dystonia, leg spasms, dysarthria, } \\
\text { blepharospasm, small steps }\end{array}$ & \\
\hline 3 & $41 / M$ & 2 (HPN) & Capiz & 32 & $\begin{array}{l}\text { Dysphagia, dysarthria, dystonia of both legs, } \\
\text { small steps, festinating gait }\end{array}$ & Hypertension \\
\hline 4 & $59 / \mathrm{M}$ & 2 (HPN) & Aklan & 52 & $\begin{array}{l}\text { Dystonia of both legs, dysarthria, blepharospasm, } \\
\text { postural instability }\end{array}$ & Hypertension \\
\hline 5 & $46 / \mathrm{M}$ & 2 (HPN,AD) & Capiz & 57 & $\begin{array}{l}\text { Generalized dystonia, neck dysarthria, } \\
\text { blepharospasm, small steps, decreased fascial } \\
\text { expression }\end{array}$ & $\begin{array}{l}\text { Hypertension } \\
\text { Anxiety Disorder }\end{array}$ \\
\hline 6 & $42 / M$ & 2 (HPN) & Antique & 38 & $\begin{array}{l}\text { Dystonia of arms and legs, neck, dysarthria, } \\
\text { blepharospasm, slow movement, festinating gait }\end{array}$ & Hypertension \\
\hline 7 & $31 / \mathrm{M}$ & 1 & Antique & 29 & $\begin{array}{l}\text { Generalized dystonia, small steps, poor balance, } \\
\text { tremor }\end{array}$ & \\
\hline
\end{tabular}


Anesthetic management of these cases started with the preoperative evaluation several days before the procedure in the Pre-anesthesia clinic. History taking included eliciting the symptoms and their onset, medications, and interventions related to XDP. Obtaining history and information was occasionally sourced from family members, caregivers, and old medical records because of the patient's dysarthria, which affected five out of the seven patients. All were either classified as ASA 1 or 2. Four of the seven patients were found to have a history of hypertension, which was being controlled by antihypertensives such as losartan, telmisartan, or amlodipine. There were no other significant systemic diseases.

Assessment for the risk of aspiration and difficult airway was done - none of the patients presented with obstructed breathing nor stridor (as seen in laryngeal adductor dystonia). Six of the seven patients have Mallampati scores of 1 . One patient had difficulty with opening and closing the jaw, and another reported dysphagia. However, it was determined that a supraglottic airway device could likely be inserted as the need arises, in all patients, even with their head fixed in a stereotactic head frame.

Preoperative laboratory tests included CBC, PT, PTT, INR, chest X-ray, and ECG. The preoperative anesthesia consults were comprehensive and ensured that patients were made aware of the technical part of the anesthesia management. The plan of being aroused from anesthesia at least twice and being awake during most of the first stage of the operation was explained to the patients to prepare them psychologically for the procedure. As with the surgery, the patients also consented to the planned anesthetic management as offered by our team.

The next active role of the anesthesiologist was on the day before surgery when a pre-procedural magnetic resonance imaging was done to map out targeted globus pallidus internal (GPi) nuclei where the electrodes were to be placed. Considering that the patients were in a state of involuntary movement and the sensitivity of the MRI machine to such movements, it was the task of the anesthesiologist to keep the patients still during the 1 hour MRI imaging procedure. In all cases, anesthesia during the MRI was maintained with target controlled infusion (TCI) of propofol at 1 to $2 \mathrm{ug} / \mathrm{mL}$, which were sufficient to control the dystonic movements and/ or tremors while maintaining a sedated but spontaneously breathing patient. MRI compatible multiparameter patient monitor was used for all cases. The actual bilateral DBS procedure was scheduled the following day. A standard preoperative fasting regimen was implemented. All patients were asked to be "off" of any dopaminergic medications.

On the morning of the surgery, in the patient's room, a stereotactic Leksell head frame was fixed to the patient's skull while awake under local anesthesia. The patients were then brought to Radiology for stereotactic localization of the coordinates with a CT SCAN of the head. Of the seven patients, six were referred to anesthesia for sedation during CT SCAN because of poor images taken when the patients were awake and vigorously moving. A bolus of propofol at a low dose of $1 \mathrm{mg} / \mathrm{kg}$ IV, titrated to effect, was found sufficient to put the patients to sleep and get acceptable images.

After the CT SCAN, the patients were transferred awake to the operating room. The patients were placed in a slight head up supine sniffing position. The stereotactic head frame was fixed to the operating room table. The legs were slightly flexed with pillows under the knees. Intermittent compressive devices were also attached to both legs. Patients were asked if they were comfortable with their position. Bair Hugger was used to keep them warm. Standard ASA monitoring equipment such as NIBP (non-invasive blood pressure), pulse oximetry, capnography, and ECG were attached to the patients. In addition, a Bispectral Index (BIS) monitoring system with a Quatro sensor pad was affixed to the patients to monitor the level of sedation throughout. Oxygen was administered to the patients at a rate of 2 liters per minute using the SENTRI ETCO ${ }_{2}$ adult nasal cannula with the $\mathrm{CO}_{2}$ monitoring line. Infusion of remifentanil was initiated at 1 to $2 \mathrm{ng} / \mathrm{mL}$, followed by infusion of propofol at 1 to $2 \mathrm{ug} / \mathrm{mL}$, both using TCI pumps. Once BIS value was between 70-80, a Foley catheter F-14 was inserted, and a radial arterial line was attached. A BIS value of 7080 means the patient was under moderate sedation, which corresponds to the Richmond Agitation Sedation Score (RASS) of -3. A complete scalp nerve block (SB) was done using a local anesthetic solution of ropivacaine $0.2 \%$ and lidocaine $1 \%$ with epinephrine 1:200,000. Six nerves were blocked on both sides to do a complete scalp block; the supraorbital and supratrochlear nerves, auriculotemporal nerve, zygomaticotemporal nerve greater occipital nerve, and lesser occipital nerve. A deeper plane of anesthesia was used during these early parts of the operation since these were painful and did not involve any testing.

Surgical preparation was done. Transparent plastic drapes were used, making sure the patient's face was not obscured. A Burr hole, first on one side, was created in the cranium. After the Burr hole, propofol and remifentanil infusions were turned off to awaken the patient bringing the BIS value of 100. An electrode was inserted through the burr hole initially $15 \mathrm{~mm}$ above the target Gpi nuclei and advanced deeper in $0.5 \mathrm{~mm}$ increments until it reached the target. At this stage, typically around 5-10 minutes from turning off the infusions, the patient should be fully awake, and cooperative, to facilitate accurate intraoperative neurophysiologic microelectrode recording (MER) done by the neurophysiologist. MER is an electrophysiologic technique used to fine-tune localization of the target nuclei, which can be determined based on their unique firing patterns. Once the electrode was in place, macro stimulation testing followed to allow the team to briefly activate the implanted electrode to confirm clinical improvement and detect any adverse side effects if stimulated. After confirmation of the electrode placement, the patients were again put to sleep, re-starting the propofol and remifentanil infusions until 
BIS value of 70-80 and the neurosurgeon proceeded with the placement of the second electrode on the opposite side of the cranium. MER and macro stimulation testing again followed this after tapering off of the anesthetic agents to an awake BIS value of 100. In instances of increasing blood pressure, expected as the patients woke up, incremental doses of nicardipine were given to keep the patients normotensive.

The second stage of DBS surgery was done under general anesthesia. This stage involved maneuvers expected to be painful. It involved the tunneling of the extension cable of the electrodes subcutaneously from the cranial area along the neck to the infraclavicular area on the right side, where they were connected to the programmable implantable pulse generator (IPG). The IPG was placed in a subcutaneous pocket created in the right subclavicular area. Induction of general anesthesia commenced with propofol $2.5 \mathrm{mg} /$ $\mathrm{kg}$ IV and rocuronium $0.6 \mathrm{mg} / \mathrm{kg}$ IV. In all the patients, an appropriate size supraglottic airway (SGA) device was used. General anesthesia was maintained with sevoflurane inhalation and remifentanil infusion. A BIS value of 40 to 60 was maintained during this stage of the procedure. It is the recommended BIS range appropriate for general anesthesia.

The average total surgical time of DBS surgery was 7 hours $(\mathrm{SD}=1.6)$, inclusive of the second stage under general anesthesia, averaging 1 hour. There was minimal blood loss. Intraoperatively, 3 patients had episodes of hypertension, which were controlled by intravenous nicardipine drip. None had an intraoperative seizure, confusion, or intracranial hemorrhage. All seven (7) patients' surgeries were completed as planned. All patients were conscious, communicative, and oriented within 5 minutes from the end of the DBS procedure. No patient complained of discomfort or recalled intraoperative pain. Adequate postoperative analgesia was achieved with intravenous parecoxib $40 \mathrm{mg}$ and paracetamol $1 \mathrm{gram}$. Antiemetics like the serotonin-receptor antagonist ondansetron was likewise given before the end of surgery. These made possible the performance of an immediate comprehensive postoperative neurological examination. No intraoperative imaging was done; however, a CT scan on the $1^{\text {st }}$ postoperative day was performed. Proper positioning of the electrodes was confirmed, and no hemorrhage, edema, or pneumocephalus was detected for all cases.

One multidisciplinary team consisting of a neurosurgeon, a movement disorder neurologist, a neurophysiologist, a neuropsychiatrist, and a neuroanesthesiologist attended to all patients. The surgical technique and anesthetic regimen were consistent for all 7 cases. The patients and relatives noted immediate improvements from Day 1 even before the stimulation was started. The average total hospital stay was five days. Patients were asked to come back 3-4 weeks after surgery for the first DBS programming. No surgical complications such as seizures, headache, confusion, nor other neurological or neuropsychological changes were reported. As of this writing, DBS surgery remarkedly relieved them of their symptoms and has changed their quality of life. None of the cases required replacement or repositioning of leads, IPG, or extension wires.

\section{DISCUSSION}

No permanent cure has been effective for XDP or Lubag disease. Pharmacological treatment offers only partial and temporary symptomatic relief. DBS, a proven surgical option for medically-refractory Parkinson's disease (PD) and generalized dystonia, provides a viable and promising treatment for XDP. ${ }^{4,7,9,10}$ It involves surgical placement of electrodes on a precise area of the brain like the GPi nuclei. In a recent review, it has been said that pallidal DBS has resulted in more consistent improvement of hyperkinetic than hypokinetic movements in patients with both dystonia and parkinsonism features. ${ }^{11}$ Local data showed that it has low perioperative morbidity and effective treatment of dystonia in the first 12 months, however, the progression of parkinsonism was still observed in some cases. ${ }^{10}$

Currently, there is no consensus on the best anesthetic technique for DBS, more so for DBS on XDP patients. Published literature reveals that anesthetic protocols vary by institutions and neurosurgeons. Patients, therefore, may receive anesthesia care in the form of monitored anesthesia care with local anesthesia, conscious sedation and general anesthesia. ${ }^{12,13,14}$ Comparison of anesthesia technique and outcomes with other series is not an objective of this paper and would probably be inappropriate due to the limited experience at present.

In our experience, all patients who underwent DBS were given BIS guided conscious sedation with low dose propofol and remifentanil infusion with scalp block at the start of the procedure then shifted to awake monitored anesthesia care during electrode placement, neuromonitoring, and testing. Patients were again sedated for the contralateral burr hole and awakened before testing. All were put under general anesthesia during the placement of the IPG in the infraclavicular area. All operations were safely completed by the same anesthesia method. All patients had successful localization, and insertion of the DBS electrode and discharged improved. Contributory to the success of this series is careful patient selection and preparation.

The preoperative visit allows for the retrieval of valuable information, including comorbidities, airway evaluation, and establishing patient rapport. Obtaining history and information was challenging for some patients with dysarthria as lower facial, oromandibular, and lingual dystonia are standard features of $\mathrm{XDP}^{1,15}$ and thus were sourced from patients' relatives and caregivers. Malnutrition and aspiration pneumonia are common in these patients due to swallowing dysfunctions. However, cough and respiratory reflexes are usually intact. ${ }^{15}$ Patients should be assessed for their willingness and ability to tolerate being awake or just lightly sedated most of the time during a potentially lengthy procedure like DBS, both physically and psychologically. 
The steps of the procedures were carefully explained to them. Reassurance was given. Some patients may request for sedation during diagnostic imaging studies such as cranial CT scan and MRI if they were claustrophobic or with severe tremors. ${ }^{12} \mathrm{XDP}$ patients for DBS are relatively younger than PD patients and will more likely have the ability to cooperate during the awake stages of the surgery. Our set of patients was mostly within the usual age of presentation of XDP at the third to fourth decade of life and treated early in their disease in that their dystonia has yet to involve the trunk. ${ }^{1}$ Truncal dystonia with hyperextension or arching, rotation, and lateral bending may present difficulties in maintaining a still patient in the operating table for long periods.

Anxiety during the procedure can lead to hypertension, which is a significant risk factor for intracranial hemorrhage (ICH). ${ }^{16} \mathrm{ICH}$ is a devastating complication of DBS that occurs in 0.6 to 3.3 percent. ${ }^{17}$ Unfortunately, benzodiazepines like midazolam are avoided as anxiolytic premedication as these sedatives, even on small amounts, can abolish MER and interfere with stimulation testing. ${ }^{12}$ This is the reason why we do not consider a completely awake, or just with local anesthesia, for DBS in our institution, despite it being done as reported in the literature. ${ }^{5,6}$ Patients who were taking antihypertensive medications preoperatively were asked to continue their medications. These included the angiotensin-converting enzyme (ACE) inhibitors and angiotensin receptor blockers (ARBs) up to the day of DBS surgery. In a retrospective study of 136 patients who underwent awake DBS surgery in one center, withholding $\mathrm{ACE}$ inhibitors and $\mathrm{ARBs}$ was an independent risk factor for intraoperative hypertension. The same risk factors determined the need to use aggressive antihypertensive therapy during the procedure. ${ }^{18}$ Patients for DBS have to be in a "drugoff" state to elicit intraoperative mapping and clinical testing. Preoperative drugs used for the treatment of motor symptoms should be stopped on the night before surgery.

Airway assessment is particularly essential as airway access and management in a patient with the head fixed with a stereotactic Leksell frame may be very difficult, if not impossible. Airway compromise may occur in 1.6 to 2.2 percent of patients undergoing DBS. ${ }^{12}$ This may occur anytime during surgery, especially when airway patency is lost during excessive sedation. Hence a plan for urgent airway management is always formulated. Rescue airway devices like a supraglottic airway, which can be inserted with the operator facing the patient and less time consuming than using direct or even video laryngoscopy, and the equipment necessary to remove the frame, must be prepared. ${ }^{19,20}$

Intraoperatively, the goal of anesthesia is to provide patients comfort by providing analgesia and sedation with minimal respiratory depression, optimize surgical condition with a slack brain and motionless patient, ensure hemodynamic stability, and facilitate intraoperative neuromonitoring with use of MER and macro stimulation testing for accurate placement of the electrodes.
Standard physiologic monitors like ECG, NIBP, pulse oximeter, temperature probe are attached to the patient. Monitoring exhaled carbon dioxide monitoring is particularly helpful in both airway vigilance and prevention of cerebral edema; thus, we utilize SENTRI ETCO adult nasal cannula for both oxygen supplementation and $\mathrm{EtCO}_{2}$ sampling in a non-intubated patient. Radial arterial lines are also placed for a minute by minute hemodynamic monitoring and control. Hypertension, as mentioned earlier, can lead to devastating complications and must be controlled before the insertion of the electrode to prevent intracranial hemorrhages. In most cases, when it occurs, nicardipine infusion promptly lowers the blood pressure without affecting physiologic testing (in contrast with beta-blockers). It is recommended that we maintain a systolic blood pressure $<130$ $\mathrm{mmHg}$ or a $20 \%$ increase of the patient's usual range. ${ }^{21}$

The initial steps of the procedure are particularly painful, including the scalp block of the six nerves on both sides of the skull, the insertion of a radial arterial line, the placement of the foley catheter and the burr holes. During this stage, the patient is sedated with low dose target-controlled infusions of propofol and remifentanil, both of which have the desirable properties of rapid onset and rapid offset. Patients wake up from propofol clear-headed. In both Parkinson's disease and dystonia, successful MERs have been performed with the target plasma concentrations that we used in our TCIs. With more confidence in our technique, we may consider lowering the propofol dose in the future. In Parkinson's patients, lowerthan-average propofol doses are needed than is predicted by TCI models, given the neurodegenerative disease. Raz et al. reported successful target localization of nuclei using low dose $(25$ to $50 \mathrm{mcg} / \mathrm{kg} / \mathrm{min}$ ) propofol infusion. His study showed that although the neuronal activity was affected, it returned to baseline shortly after propofol administration was stopped. ${ }^{22}$ In XDP, the DBS target, Gpi nucleus, is susceptible to even small doses of anesthetic agents due to a large GABAergic input from the striatum.

In some centers, these particular concerns led to the use of non-GABAergic drugs such as dexmedetomidine and ketamine (in pediatric patients) in DBS..$^{14,23,24}$ Reports have shown that continuous infusion of dexmedetomidine with or without intermittent small doses of propofol was equally satisfactory as sedative regimens. ${ }^{25}$ However, we preferred propofol as it was faster to adjust the level of sedation, and maintaining a motionless surgical field, than with changing the infusion rate of dexmedetomidine. Another particular benefit of using propofol versus dexmedetomidine is the possibility of amnesia for the former. In a retrospective questionnaire interview of patients who underwent awake/ MAC (monitored anesthesia care) with local anesthesia DBS electrode insertion, almost all recalled physical pain and psychological suffering during the procedure. ${ }^{26}$ We believe that patients will appreciate the degree of unconsciousness and, thus, the amnesia, for parts of the procedure that are distressing such as postural pain by fixation for hours, the 
unpleasant feelings of manipulations in the head, the stress from unfamiliar surroundings and the disturbing noises of drilling and suction.

Remifentanil is an opioid receptor agonist with excellent analgesic properties but minimal effect on MER; hence, it is ideal for DBS surgery. In a retrospective study using propofol and remifentanil, wake-up time varied among patients and types of operation, but a median wake-up time of 9 minutes was achieved. However, more delayed arousal of up to 36 minutes was seen in patients with dystonia undergoing DBS surgery. ${ }^{27}$ The rate of remifentanil infusion can be adjusted independently of propofol to improve patient comfort or alter a patient's breathing rate during spontaneous ventilation. Some reports suggested that remifentanil at $0.01 \mathrm{mg} / \mathrm{kg} / \mathrm{min}$ to $0.05 \mathrm{mg} / \mathrm{kg} / \mathrm{min}$ or (dexmedetomidine, if used) can be continued during the awake period. ${ }^{28}$

In all our patients, aside from using it at a very low dose with BIS guidance, the infusion is discontinued immediately after the burr hole surgery. This gives sufficient time to awaken the patients before intraoperative neuromonitoring to offset concerns of its effect in decreasing nuclei neuronal activity at high doses.

\section{BIS}

The Bispectral index (BIS) is a dimensionless numerical scale for measuring cerebral electrical activity and depth of anesthesia. Its value is a number between 0 to 100 , where 0 represents no detectable brain electrical activity, and 100 represents an awake state. The use of BIS during DBS to guide the dose of the propofol and remifentanil infusion has certain advantages. It prevents over-sedation of patients and consumption of too much anesthetics, which can put the patient at risk of hypotension and respiratory compromise. Likewise, it shortens recovery time from asleep to awake state, which is desirable in DBS surgery while avoiding light anesthesia that may increase surgical complications with sudden movements or with hypertension with anxiety and pain. Some contend that BIS does not seem to correlate well with clinical assessment of patients with movement disorders who often have more EMG (electromyography) activity than those without movement disorders. ${ }^{29}$ But since we are still in the early part of our learning curve with this analgosedative technique, we cannot and did not rely entirely on clinical assessment. BIS guidance afforded rapid and smooth shifting of levels of anesthesia.

During propofol-remifentanil infusion in the early part of the first stage of surgery, the BIS level was maintained between 70-80. After the painful part, infusions were stopped to allow the patient to wake up with BIS value going up to a baseline of 100 . The use of BIS confirms the patient was awake and ensured the accuracy of placement of electrodes through MER and stimulation testing. In stage 2 of surgery, when patients were placed under general anesthesia, BIS value was maintained between 40-60, an appropriate value for general anesthesia, at this painful stage.
SB

Although the brain itself has no pain receptors, the scalp, periosteum, and meninges all have pain receptors. Sufficient local anesthesia is mandatory to minimize pain and hypertensive episodes during DBS. There are two options commonly described in the literature. One option was to use subcutaneous infiltration of local anesthetics on the burr holes and pin sites. A second option is to do a scalp block (SB). The supraorbital, supratrochlear, auriculotemporal, zygomaticotemporal, lesser occipital, and greater occipital nerves on both sides of the cranium were blocked using long-acting local anesthetic like ropivacaine $0.25 \%$ with epinephrine 1:200,000, which typically provides 6 to 8 hours of analgesia. The use of SB also lessens the need to use higher doses of propofol and remifentanil infusion, making the technique balanced anesthesia. Krauss et al. retrospectively analyzed intraoperative cardiovascular parameters and perioperative medication in 47 patients $(\mathrm{LA}=29, \mathrm{SB}=18)$ undergoing DBS. Primary study endpoints were intraoperative systolic blood pressure and heart. Secondary endpoints were the use of intraoperative antihypertensives. Patients who had $\mathrm{SB}$ showed lower mean systolic BP and heart rate compared with patients who were on LA. Patients who had LA required more antihypertensive medication to stabilize $\mathrm{BP} .^{30}$

\section{IPG Insertion}

The implantation of the IPG is painful, hence, it was performed under general anesthesia. It is often placed in the right infraclavicular area. After the insertion of both right and left electrodes, a long cable connecting these electrodes and the IPG is tunneled subcutaneously from the burr hole through the neck to the IPG. For this stage, the patient is placed under general anesthesia using either an endotracheal tube or a supraglottic airway device. In all our patients, a supraglottic airway device was chosen. The advantages of a supraglottic airway device include less coughing, bucking, and hemodynamic changes compared to awakening with an endotracheal tube in place. ${ }^{19}$ It is also easier to insert, remove, and reinsert without changing the position of a patient's head. ${ }^{20}$

\section{CONCLUSION}

Different institutions have developed their anesthetic protocols of performing DBS procedure for XDP patients, depending upon neurosurgeons' need and patients' status. In our institution, we use BIS guided conscious sedation with low dose propofol and remifentanil TCI infusions with SB during the early painful part, awake with monitored anesthesia care during the placement of electrodes to allow accurate MER and macro stimulation testing and general anesthesia during the insertion of infraclavicular IPG with excellent results. Our experience highlights the need for proactive participation of the neuroanesthesiologist in all the stages of the DBS surgery. It likewise emphasizes the need to 
be prepared to utilize the whole gamut of anesthesia skillsconscious sedation, scalp blocks, monitored anesthesia care, and general anesthesia. In our early experience, all operations were completed safely using the described anesthesia method, with successful localization, and insertion of the DBS electrode. We have to continually document the outcomes of locally performed DBS procedures with particular focus on the anesthetic technique to allow us to develop protocols for this type of patient. Our early experience was limited to a few cases, and thus we were unable to make significant conclusions and recommendations. It is, however, recommended to form a dedicated DBS team in every institution to overcome the learning curve, standardize techniques, and achieve excellent outcomes.

\section{Statement of Authorship}

All authors participated in the conceptualization, data collection and analysis. All authors have approved the final version submitted.

\section{Author Disclosure}

All authors declared no conflicts of interest.

\section{Funding Source}

None.

\section{REFERENCES}

1. Evidente VG, Advincula J, Esteban R, Pasco P, Alfon JA, Natividad FF, et al. Phenomenology of "Lubag" or X-linked dystonia-parkinsonism. Mov Disord. 2002; 17(6):1271-7.

2. Jamora RD, Teleg RA, Cordero CP, Villareal-Jordan RF, Lee LV, Pasco PM. Levodopa+ carbidopa in X-linked dystonia parkinsonism (XDP/ DYT3/Lubag): a randomized, double-blind, placebo-controlled trial. Acta Med Philipp. 2018; 52:510-4.

3. Evidente VG, Lyons MK, Wheeler M, Hillman R, Helepolelei L, Beynen F, et al. First case of X-linked dystonia-parkinsonism ("Lubag") to demonstrate a response to bilateral pallidal stimulation. Mov Disord. 2007; 22(12):1790-3.

4. Wadia PM, Lim SY, Lozano AM, Adams JR, Poon YY, Torres Diaz CV, et al. Bilateral pallidal stimulation for $\mathrm{x}$-linked dystonia parkinsonism. Arch Neurol. 2010; 67(8):1012-5.

5. Aguilar JA, Vesagas TS, Jamora RD, Teleg RA, Ledesma L, Rosales $\mathrm{RL}$, et al. The promise of deep brain stimulation in X-linked dystonia parkinsonism. Int J Neurosci. 2011; 121(supp11):57-63.

6. Ohshita N, Tanaka K, Sakai Y, Katayama T, Tomiyama Y, Ohsita S, et al. Anesthesia for deep brain stimulation in a patient with X-linked dystonia-parkinsonism/Lubag disease. J Med Invest. 2013; 60(12):146-8.

7. Brüggemann N, Domingo A, Rasche D, Moll CKE, Rosales RL, Jamora RDG, et al. Association of Pallidal Neurostimulation and Outcome Predictors With X-linked Dystonia Parkinsonism. JAMA Neurol. 2019; 76(2):211-6.

8. De Roxas RC, Jamora RDG. Cost-Analysis of the Different Treatment Modalities in X-Linked Dystonia-Parkinsonism. Front Neurol. 2019;10:500.

9. Diestro JDB, Vesagas TS, Teleg RA, Aguilar JA, Anlacan JP, Jamora RDG. Deep brain stimulation for Parkinson disease in the Philippines: outcomes of the Philippine Movement Disorder Surgery Center. World Neurosurg. 2018; 115:e650-8.

10. Abejero JE, Jamora RD, Vesagas TS, Teleg RA, Rosales RL, Anlacan JP, Velasquez MS, Aguilar JA. Long-term outcomes of pallidal deep brain stimulation in $\mathrm{X}$-linked dystonia parkinsonism (XDP): Up to 84 months follow-up and review of literature. Parkinsonism Relat Disord. 2019; 60:81-6.

11. Bragg DC, Sharma N, Ozelius LJ. X-linked dystonia-parkinsonism: recent advances. Curr Opin Neurol. 2019; 32(4):604-9.

12. Venkatraghavan L, Luciano M, Manninen P. Review article: anesthetic management of patients undergoing deep brain stimulator insertion. Anesth Analg. 2010; 110(4):1138-45.

13. ScharpfDT, Sharma M, Deogaonkar M, Rezai A, Bergese SD. Practical considerations and nuances in anesthesia for patients undergoing deep brain stimulation implantation surgery. Korean J Anesthesiol. 2015; 68(4):332-9.

14. Osborn IP, Kurtis SD, Alterman RL. Functional neurosurgery: anesthetic considerations. Int Anesthesiol Clin. 2015; 53(1):39-52.

15. Song PC, Le H, Acuna P, De Guzman JKP, Sharma N, Francouer $\mathrm{TN}$, et al. Voice and swallowing dysfunction in X-linked dystonia parkinsonism. Laryngoscope. 2020; 130(1):171-7.

16. Xiaowu H, Xiufeng J, Xiaoping Z, Bin H, Laixing W, Yiqun C, et al. Risks of intracranial hemorrhage in patients with Parkinson's disease receiving deep brain stimulation and ablation. Parkinsonism Relat Disord. 2010; 16(2):96-100.

17. Khatib R, Ebrahim Z, Rezai A, Cata JP, Boulis NM, John Doyle D, et al. Perioperative events during deep brain stimulation: the experience at Cleveland clinic. J Neurosurg Anesthesiol. 2008; 20(1):36-40.

18. Rajan S, Deogaonkar M, Kaw R, Nada EM, Hernandez AV, Ebrahim $Z$, Avitsian R. Factors predicting incremental administration of antihypertensive boluses during deep brain stimulator placement for Parkinson's disease. J Clin Neurosci. 2014; 21(10):1790-5.

19. Brimacombe J. The advantage of the LMA over the tracheal tube or facemask: a meta-analysis. Can J Anaesth. 1995; 42(11):1017-23.

20. Brockerville M, Unger Z, Rowland NC, Sammartino F, Manninen $\mathrm{PH}$, Venkatraghavan L. Airway management with a stereotactic headframe in situ - a Mannequin Study. J Neurosurg Anesthesiol. 2018; 30(1):44-8

21. Jian M, Li X,Wang A , Zhang L, Han R, Gelb AW. Flurbiprofen and hypertension but not hydroxyethyl starch are associated with postcraniotomy intracranial haematoma requiring surgery. Br J Anaesth. 2014; 113(5):832-9.

22. Raz A, Eimerl D, Zaidel A, Bergman H, Israel Z. Propofol decreases neuronal population spiking activity in the subthalamic nucleus of Parkinsonian patients. Anesth Analg. 2010; 111(5):1285-9.

23. Rozet I, Muangman S, Vavilala MS, Lee LA, Souter MJ, Domino KJ, et al. Clinical experience with dexmedetomidine for implantation of deep brain stimulators in Parkinson's disease. Anesth Analg. 2006; 103(5):1224-8.

24. Hippard HK, Watcha M, Stocco AJ, Curry D. Preservation of microelectrode recordings with non-GABAergic drugs during deep brain stimulator placement in children. J Neurosurg Pediatr. 2014; 14(3):279-86

25. Chui J, Alimiri R, Parrent A, Craen RA. The Effects of Intraoperative Sedation on Surgical Outcomes of Deep Brain Stimulation Surgery. Can J Neurol Sci. 2018; 45(2):168-75.

26. Mulroy E, Robertson N, Macdonald L, Bok A, Simpson M. Patients' perioperative experience of awake deep-brain stimulation for Parkinson disease. World Neurosurg. 2017; 105:526-8.

27. Trombetta C, Deogaonkar A, Deogaonkar M, Ebrahim Z, Rezai A, Machado A, et al. Delayed awakening in dystonia patients undergoing deep brain stimulation surgery. J Clin Neurosci. 2010;17(7):865-8.

28. Grant R, Gruenbaum SE, Gerrard J. Anaesthesia for deep brain stimulation: a review. Curr Opin Anaesthesiol. 2015; 28(5):505-10.

29. Schulz U, Keh D, Barner C, Kaisers U, Boemke W. Bispectral index monitoring does not improve anesthesia performance in patients with movement disorders undergoing deep brain stimulating electrode implantation. Anesth Analg. 2007; 104(6):1481-7.

30. Krauss P, Marahori NA, Oertel MF, Barth F, Stieglitz LH. Better hemodynamics and less antihypertensive medication: comparison of scalp block and local infiltration anesthesia for skull-pin placement in awake deep brain stimulation surgery. World Neurosurg. 2018; 120:e991-9. 